EUROPEAN ORGANIZATION FOR NUCLEAR RESEARCH

CERN - AB Division

CERN-AB-2003-015 ABP

\title{
Adiabatic Beam Trapping in Stable Islands of Transverse Phase Space: Measurement Results at CERN Proton Synchrotron
}

\author{
R. Cappi, M. Giovannozzi, M. Martini, E. Métral, \\ G. Métral, R. Steerenberg, CERN, Geneva, Switzerland, \\ A. S. Müller, ISS, Forschungszentrum Karlsruhe, Germany.
}

\begin{abstract}
Recently a novel approach to the problem of multi-turn extraction was proposed. It consists of splitting the beam by adiabatic capture inside stable islands created in the transverse phase space by sextupoles and octupoles. Numerical simulations indicate that such a technique should be feasible and potentially superior to the method presently used at the CERN Proton Synchrotron. During 2002, intense efforts were devoted to the experimental verification of this newly proposed extraction mode. Finally, beam capture into the islands was observed. In this paper, the extraction principle is briefly reviewed and the experimental results are presented and discussed in detail.
\end{abstract}

Presented at PAC 2003, Portland, Oregon, USA
from 12 to 16 May 2003

Geneva, Switzerland

22 May 2003 


\title{
ADIABATIC BEAM TRAPPING IN STABLE ISLANDS OF TRANSVERSE PHASE SPACE: MEASUREMENT RESULTS AT CERN PROTON SYNCHROTRON
}

\author{
R. Cappi, M. Giovannozzi, M. Martini, E. Métral, G. Métral, R. Steerenberg, CERN, Geneva, Switzerland \\ A.-S. Müller, ISS, Forschungszentrum Karlsruhe, Germany
}

\section{Abstract}

Recently a novel approach to the problem of multi-turn extraction was proposed. It consists of splitting the beam by adiabatic capture inside stable islands created in the transverse phase space by sextupoles and octupoles. Numerical simulations indicate that such a technique should be feasible and potentially superior to the method presently used at the CERN Proton Synchrotron. During 2002, intense efforts were devoted to the experimental verification of this newly proposed extraction mode. Finally, beam capture into the islands was observed. In this paper, the extraction principle is briefly reviewed and the experimental results are presented and discussed in detail.

\section{INTRODUCTION}

With the approval of the CERN Neutrino to Gran Sasso Project [1], efforts were devoted to the feasibility study of an intensity upgrade of the Proton Synchrotron (PS) and Super Proton Synchrotron (SPS) complex [2]. A delicate point in the present scheme for the beam generation is the multi-turn extraction from PS to SPS, the so-called Continuous Transfer (CT) [3]. Due to the difference in circumference, $C_{\mathrm{SPS}}=11 C_{\mathrm{PS}}$, and given the constraint of minimising the SPS filling time, the beam is extracted from the PS over five turns in two consecutive cycles. This is obtained by means of an electrostatic septum, used to slice the beam, and a proper choice of the horizontal tune (6.25) (see Refs. [3, 4] for more details). The main drawbacks of this technique are the intrinsic losses on the electrostatic septum and the poor betatron matching of the five slices [4], which in turn might transfer into injection losses into the SPS.

Recently, an alternative method was proposed, where the beam is split in the transverse phase space by means of adiabatic capture inside stable islands [5, 6] (see Fig. 1 for typical simulation results). The new technique would allow overcoming the bottlenecks of the present extraction mode. Not only, beam losses are reduced to almost zero, but also the phase space matching is highly improved (see Ref. [4] for a comparative analysis of the two approaches).

Following the positive results of the numerical simulations, further simulation studies were carried out to get more insight in the capture process [7], as well as experimental measurements to asses the feasibility of this new extraction mode.

\section{MEASUREMENT CAMPAIGN}

\section{Machine and Instrumentation Modifications}

To generate the stable islands used to capture the beam, nonlinear elements, such as sextupoles and octupoles, are needed. To this aim two sextupoles were installed in the ring, as well as two octupoles: elements of the same type are connected in series to provide the necessary strength and each pair is powered independently.

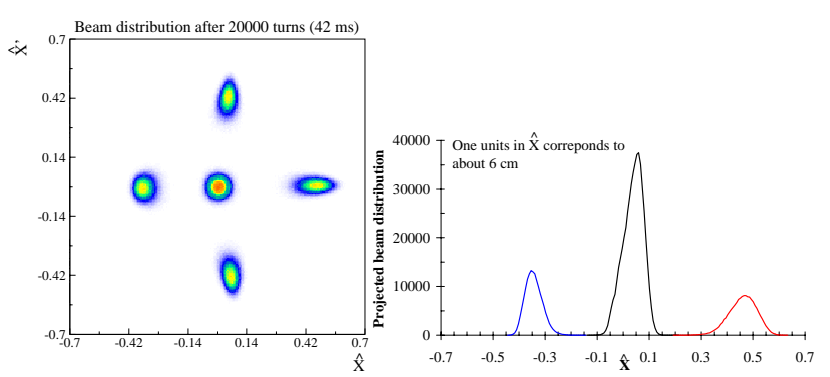

Figure 1: Results of the numerical simulations for the adiabatic capture inside stable islands (left: final pahse space distribution, right: projected beam distribution onto horizontal axis).

Two types of measurements are needed, namely phase space measurement and beam profile measurement during the various stages of the beam capture. The first one requires a multi-turn measurement of the beam position using two beam position monitors $90^{\circ}$ apart in phase space [8]. A new acquisition system, based on a fast digitiser, was developed $[9,10]$. The beam profile measurement was obtained with a flying wire scanner device [11] currently available in the PS ring. A schematic layout of the PS ring including the newly installed elements as well as other key devices for the adiabatic beam capture tests described in this paper is shown in Fig. 2.

\section{Phase Space Measurement}

The new extraction mode requires a precise control of the islands' position and size. Hence, a good knowledge of the phase space structure is of uttermost importance.

The technique used is the standard one, i.e. the beam trajectory is perturbed by means of a kicker magnet (notably the one normally used to fast extract the beam) and betatron oscillations are observed on two pickups $90^{\circ}$ apart. To overcome some difficulties due to the islands' phase 


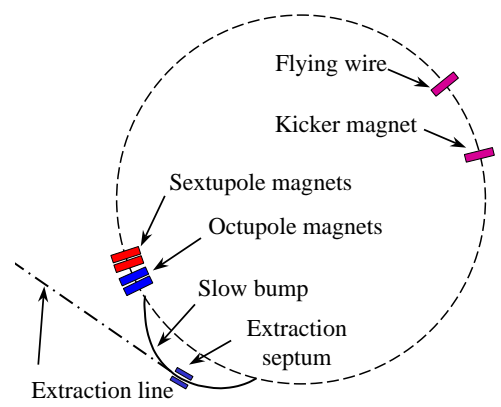

Figure 2: Layout of the PS machine including the key elements for the test of adiabatic capture. Other elements (not shown here) are involved in the final stage of the five-turn extraction.

at the kicker location, two kicks separated by three turns, which is the minimum delay due to hardware limitations, were used, thus allowing to scan along the diagonal in phase space. During the measurements the special sextupoles and octupoles were set to their computed values, i.e. $K_{2}=1.04 \mathrm{~m}^{-2}$ and $K_{3}=127.95 \mathrm{~m}^{-3}$, where $K_{i}=L /\left(B_{0} \rho\right) \partial^{i} B_{y} / \partial x^{i}$ are the sextupolar and octupolar integrated gradients. These measurements are strongly affected by beam de-coherence. In fact, as the beam position monitors measure the centre of gravity of the beam charge distribution, the detected signal fades out after some turns. Special care was taken to minimise such an effect. In particular, a low-intensity (about $6 \times 10^{11}$ protons), single-bunch beam was chosen to have small emittances, defined as $\epsilon_{h / v}^{*}=\beta \gamma \sigma_{h, v}^{2} / \beta_{h, v}$, namely $\epsilon^{*}{ }_{h} \approx 2 \mu \mathrm{m}$, $\epsilon^{*}{ }_{v} \approx 1.5 \mu \mathrm{m}$ and $\Delta p / p \approx 1.5 \times 10^{-3}$. This choice allows scanning better the phase space structures, also avoiding filamentation in the transverse plane, and hence signal decoherence. Furthermore, due to the plain FOFDOD structure of the PS lattice, no location with zero dispersion exists, implying that the dedicated sextupoles and octupoles have a strong chromatic effect, leading to a measured value of $\xi_{h}=Q^{\prime}{ }_{h} / Q_{h} \approx 1.7$ and $\xi_{v} \approx 0.6$. After a careful tuning, the chromaticity was reduced to $\xi_{h} \approx 0.1$ and $\xi_{v} \approx 0.9$ (see Ref. [12] for more details on this point). Also, the rfvoltage was decreased to reduce $\Delta p / p$ to about $0.4 \times 10^{-3}$. The main results of the phase space measurement campaign are shown in Fig. 3. The various plots refer to different kick amplitudes. In the first portrait, regular motion represented by circular phase space trajectories is visible and signal de-coherence is also apparent. As the the kick amplitude is further increased, fourth-fold symmetrical trajectories appear: the beam is kicked inside the stable islands of the fourth-order resonance. A rather strong signal decoherence is revealed by the curly-shaped beam trajectory, spiralling towards the origin. In principle, particles inside the islands should generate a coherent, albeit small, signal lasting over a long period. The strong de-coherence can be explained by assuming that time-dependent effects make the islands moving in phase space. This is the case when tune ripple is present or when considerable coupling between longitudinal and transverse degrees-of-freedom in- duce tune modulation via chromaticity. In fact, it turned out that the improvement of the quadrupoles power supplies made it possible to cure completely the problem.

Finally, it is worthwhile pointing out that the results shown in Fig. 3 are in reasonable agreement with the model of the PS machine and with the phase space topology assumed in the numerical simulations $[5,6]$.
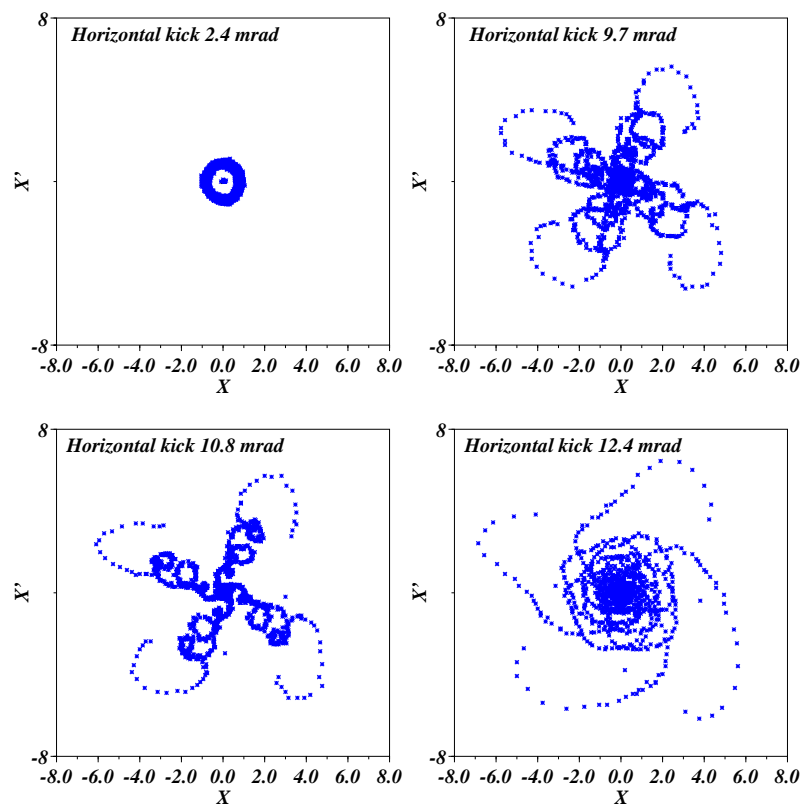

Figure 3: Horizontal normalised phase space measured with the multi-turn system (about $1.5 \times 10^{3}$ turns are plotted).

\section{Adiabatic Capture}

After having verified that the main ingredients, namely stable islands, were present in the phase space for the nominal parameters used in numerical simulations, the first tests of adiabatic capture were undertaken. To this aim, the horizontal tune was swept through the fourth-order resonance to induce and observe the trapping phenomenon. The horizontal emittance was increased $\left(\epsilon^{*}{ }_{h} \approx 12 \mu \mathrm{m}\right)$ to simulate a high-intensity beam, the other beam parameters being unchanged. The experimental conditions are shown in Fig. 4, where the horizontal tune, the strength of the nonlinear elements, and the beam intensity are plotted as a function of time. When the strength of the nonlinear elements levels out, the beam momentum is $14 \mathrm{GeV} / \mathrm{c}$ and the magnetic field is constant: the power supplies are rather slow, imposing to start ramping during acceleration. The initial value of the tune is chosen near the fourth-order resonance, then a linear change is applied, during which the capture of beam particles inside islands occurs. The final value of the tune is varied for different beam profile measurements to change the islands' separation. As far as the beam intensity is concerned, some minor losses are visible during the ramping of the nonlinear elements, while during the actual trapping no sign of losses is visible. 


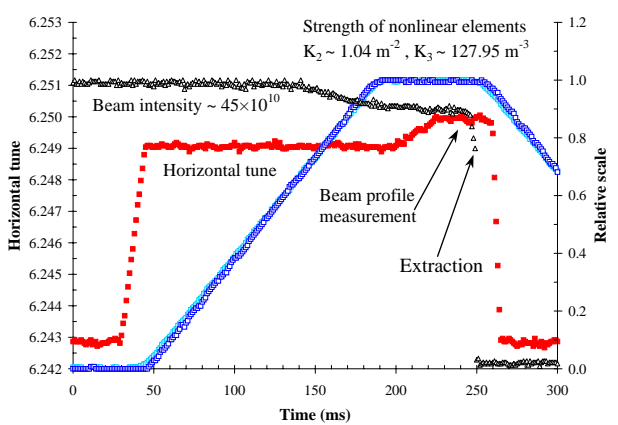

Figure 4: Experimental conditions during the tests for adiabatic capture inside stable islands.

The main results are shown in Fig. 5, where the beam profile measured by the flying wire scanner is shown for different values of the final tune.
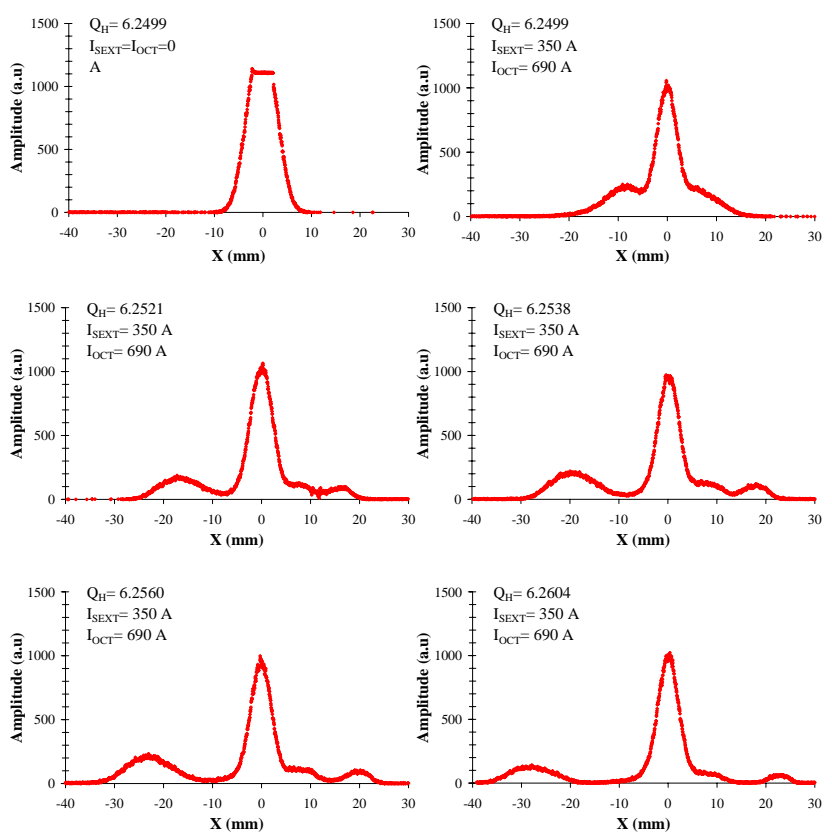

Figure 5: Horizontal beam profile measured by a flying wire scanner, for different final values of the tune. The first profile represents the initial beam distribution, when no capture occurs. The choice of the gain, optimised for the subsequent stages of adiabatic capture, explains the signal saturation.

The first profile is the reference picture: the tune is changed, but as the nonlinear elements are switched off no effect on the beam distribution is visible. The other pictures differ by the value of the final tune: the nonlinear elements are switched on and three additional peaks are clearly visible, whose separation increases as the tune is varied. One should keep in mind that, although the beam is split in five beamlets $[5,6]$, the wire scanner measures the projection onto the x-axis (see also Fig. 1), therefore, the central peak is higher than the others as it represents the superposition of three beamlets.

\section{CONCLUSIONS AND OUTLOOK}

The first tests of adiabatic beam trapping inside stable islands proved that the capture process, already observed in numerical simulations, occurs also in real machines. Beam splitting in five beamlets was detected and their separation measured as a function of the final value of the horizontal tune. Further tests are planned to study the dependence of the beam parameters on the speed of the tune variation (adiabaticity), and on sextupole and octupole strengths. Then it will be crucial testing this novel approach using higherintensity bunches as well as multi-bunch beams. Of course, actual five-turn extraction will be also tested during the 2003 experimental campaign.

\section{ACKNOWLEDGEMENTS}

We would like to thank M. E. Angoletta, J. Belleman, J. L. Gonzalez for developing and supporting the multiturn acquisition system, and M. Benedikt, M. Chanel for preparing the different beams in the PS-Booster.

\section{REFERENCES}

[1] K. Elsener (Ed.) et al., "The CERN Neutrino Beam to Gran Sasso (Conceptual Technical Design)”, CERN 98-02 (1998).

[2] R. Cappi (Ed.) et al., "Increasing proton intensity of PS and SPS", CERN-PS (AE) 2001-041 (2001).

[3] C. Bovet et al., in 1973 Particle Accelerator Conference, edited by D. W. Dupen (IEEE, New York, 1973) p. 438.

[4] R. Cappi, M. Giovannozzi, "Computation of Betatron Mismatch and Emittance Blow-up for Multi-Turn Extraction", CERN PS (AE) 2002-083 (2002).

[5] R. Cappi, M. Giovannozzi, Phys. Rev. Lett. 88, (2002) 104801.

[6] R. Cappi, M.Giovannozzi, in Eighth Particle European Accelerator Conference, edited by J. Poole and C. Petit-Jean-Genaz (Institute of Physics, UK London, 2002) p. 1250.

[7] R. Cappi, M. Giovannozzi, "Adiabatic Capture of Charged Particles in Stable Islands: a Novel Approach to Multi-Turn Extraction", these proceedings.

[8] P.Morton et al., IEEE Trans. on Nucl. Sci. 32, (1985) p. 2291.

[9] M. E. Angoletta et al., in Eighth Particle European Accelerator Conference, edited by J. Poole and C. Petit-Jean-Genaz (Institute of Physics, UK London, 2002) p. 1273.

[10] M. E. Angoletta, A.-S. Müller, in Eighth Particle European Accelerator Conference, edited by J. Poole and C. Petit-Jean-Genaz (Institute of Physics, UK London, 2002) p. 1948.

[11] C. Steinbach, M. Van Rooij, IEEE Trans. Nucl. Sci. 32, (1985) p. 1920.

[12] R. Cappi et al., "Optics Studies for the CERN Proton Synchrotron Machine: Linear and Nonlinear Modelling Using Beam Based Measurements", these proceedings. 